\title{
»Die Oper des Sagens« von Michel de Certeau
}

\author{
Ouvertüre
}

Johanna Breidenbach und Lucie Kaennel

\section{Der Autor}

Michel de Certeau (1925-1986) war ein französischer Jesuit und Geisteswissenschaftler, der sich zeit seines Lebens an Grenzen abgearbeitet hat: Er hat sie vermessen, ist ihren schmalen Linien gefolgt, die sie zwischen ein >diesseits` und ein >jenseits` ziehen, hat sie überschritten und auch erweitert. Paradigmatisch lässt sich das an derVielfalt der Disziplinen aufzeigen, in denen er zu Hause war: Theologie, Philosophie, Historiographie, Kulturwissenschaften, Psychoanalyse gehören unter anderen zu den Räumen, die er im wissenschaftlichen Betrieb der Universität für einander aufzuschliessen suchte. ${ }^{1}$ Nach einem ersten abgeschlossenen Studium in Altphilologie, klassischer Literatur, Theologie und Philosophie trat er 1950 in den Jesuitenorden ein und durchlief noch einmal die vorgesehenen philosophischen und theologischen Studien des Ordens. $^{2}$ Prägend waren für seine akademische Laufbahn nach dieser langen Ausbildung Reisen nach Südamerika sowie biographische, gesellschaftliche und kirchliche Umbrüche, die ab den 60er Jah-

\footnotetext{
1 Diese Interdisziplinarität wird oft von Certeau selbst und auch in der Sekundärliteratur als »Kunst des Wilderns» bezeichnet.

2 Luce Giard, Michel de Certeau. Ein biographisches Porträt, in: Marian Füssel (Hg.), Michel de Certeau. Geschichte - Kultur - Religion, Konstanz 2007, 21-32, 23f. Zum Leben und Werk Certeaus sei auch folgende Literatur empfohlen: François Dosse, Michel de Certeau. Le marcheur blessé, Paris 2002; Christian Delacroix/François Dosse/Patrick Garcia/Michel Trebisch (Hg.), Michel de Certeau. Les chemins d'histoire, Brüssel 2002; Luce Giard (Hg.), Le voyage mystique. Michel de Certeau, Paris 1988 (Bibliogr. der Werke von Certeau, 191-243); Claude Geffré (Hg.), Michel de Certeau ou la différence chrétienne, Paris 1991; Jeremy Ahearne, Michel de Certeau. Interpretation and Its Other, Cambridge 1995; Inigo Bocken (Hg.), Spiritual Spaces. History and Mysticism in Michel De Certeau, Leuven 2013. Zur Rezeption des Werkes von Certeau vgl. Éric Maigret, Les trois héritages de Michel de Certeau. Un projet éclaté d'analyse de la modernité, in: Annales. Histoire, sciences sociales 55 (2000), 511-549.
} 
ren eintraten und auf die er sensibel reagierte. Die akademische Anerkennung blieb ihm trotz seiner regen Forschungs- und Publikationstätigkeit lange weitgehend verwehrt; vielleicht auch, weil Grenzgänger stets etwas Verunsicherndes haben. Ab 1978 lehrte er in San Diego und befasste sich vertieft mit der amerikanischen Kultur- und Sozialwissenschaft. ${ }^{3}$ In diesem Gebiet erfolgte seine Rezeption bislang am breitesten. Schliesslich wurde er 1984, zwei Jahre vor seinem Tod, an die École des hautes études en sciences sociales berufen. $\mathrm{Zu}$ seinen Hauptwerken gehört neben der Kunst des Handelns, ${ }^{4}$ dem Schreiben der Geschichte ${ }^{5}$ und der GlaubensSchwachheit ${ }^{6}$ die Mystische Fabel, ${ }^{7}$ eine Summe von Untersuchungen zur Sprache der Mystik in der frühen Neuzeit. Das sprachtheoretische Interesse Certeaus ist sichtbar in der Mitte seines Werkes angesiedelt $^{8}$ und eben diesem verdanken wir auch seine Beschäftigung mit der Glossolalie.

3 Marian Füssel, Einleitung: Ein Denker des Anderen, in: Ders. (Hg.), Michel de Certeau, 7-19, 9 .

4 Michel de Certeau, L'invention du quotidien, Bd. 1: Arts de faire, u. Bd. 2: Habiter, cuisiner, Paris 1980. Dt. Übers. des ersten Bandes: Die Kunst des Handelns. Aus dem Französischen übersetzt von Ronald Voullié, Berlin 1988.

5 Michel de Certeau, L'écriture de l'histoire, Paris 1975; dt. Übers.: Das Schreiben der Geschichte. Aus dem Französischen übersetzt von Sylvia M. Schomburg-Scherff. Mit einem Nachwort von Roger Chartier, Frankfurt a.M./Paris 1991.

6 Michel de Certeau, La faiblesse de croire, Paris 1987; dt. Übers.: GlaubensSchwachheit. Aus dem Französischen übersetzt von Michael Lauble, Stuttgart 2009.

7 La fable mystique $\mathrm{XVI}^{\mathrm{e}}-\mathrm{XVII}^{\mathrm{e}}$ siècle, Bd. 1, Paris 1982; Bd. 2, hg. v. Luce Giard, Paris 2013. Dt. Übers. des ersten Bandes: Mystische Fabel. 16. bis 17. Jahrhundert. Aus dem Französischen übersetzt von Michael Lauble. Mit einem Nachwort von Daniel Bogner, Berlin 2010.

8 Hier sei besonders auf folgende Studien von Certeau hingewiesen: La prise de parole. Pour une nouvelle culture, Paris 1968, erweiterte Ausgabe unter dem Titel: La prise de parole et autres écrits politiques, Paris 1994; Le parler angélique, in: M. de Certeau, La fable mystique, Bd. 2 (Anm. 7), 257-286 [Originaldruck unter dem Titel: Le parler angélique. Figures pour une poétique de la langue, in: Actes sémiotiques. Documents 6:54 (1984), 7-31]; zusammen mit Dominique Julia und Jacques Revel, Une politique de la langue. La Révolution française et les patois: l'enquête de Grégoire, Paris 1975. 


\section{Der Aufsatz}

Der Glossolalie wandte er sich Ende der 70er, Anfang der 80er Jahre im Zusammenhang seines Interesses für mündliche Aussageakte zu. ${ }^{9}$ Den vorliegenden Aufsatz verfasste er 1980 als Vortrag für eine Tagung in Urbino; er erschien dann erstmals ebenfalls 1980 in der vom Pariser Centre national d'art et de culture Georges-Pompidou (dem sogenannten Centre Beaubourg) herausgegebenen Zeitschrift Traverses und wurde 2013 im zweiten Band der Fable mystique wiederabgedruckt. ${ }^{10}$ In der folgenden Zeit sammelte sich, als durch die Auflösung der Lacanschen Schule in Paris Forschungskapazitäten freigesetzt wurden, eine informelle Gruppe von Forschern unterschiedlicher Fachgebiete (der Psychoanalyse, der Linguistik, der Medizin und Literaturtheorie) um ihrem Interesse an der Glossolalie weiter nachzugehen. ${ }^{11}$ Sie beschäftigte sich dabei schwerpunktmässig mit den Forschungen zur Glossolalie Ende des 19., Anfang des 20. Jahrhunderts, so wie es auch in Certeaus Aufsatz der Fall ist. Eine weitere Tagung wurde ins Leben gerufen und eine breite Publikation unter der Regie von Certeau und dem Linguisten Jean-Jacques Courtine geplant. Sie konnte erst nach seinem Tod erscheinen und enthält neben einer Hommage an Certeau gleichsam das Kompendium der Forschungsergebnisse dieser Gruppe. ${ }^{12}$ Inzwischen liegt auch eine englische Übersetzung des Aufsatzes vor, die 1996 in der Zeitschrift Representations erschienen ist und von Daniel Rosenberg besorgt wurde. $^{13}$

In der "Oper des Sagens" untersucht Certeau in acht Abschnitten das Phänomen der Glossolalie als ein Phänomen der Inszenierung von Sprache, die den gegenständlichen Gebrauch von Worten immer wieder durchbricht und verfremdet. Dabei kann die Motivation dieser Inszenierung religiös oder auch anders gefärbt sein; es macht das Denken von Certeau aus, dass er seine Analysen mit grosser begrifflicher Unvoreingenommenheit vorträgt und deren Phänomen-

\footnotetext{
9 Vgl. auch zum Folgenden Dosse, Michel de Certeau, 312.

${ }^{10}$ Michel de Certeau, Utopies vocales: glossolalies, in: Traverses 20 (1980), 26-37; wieder abgedruckt unter dem Titel: L'opéra du dire: glossolalies, in: M. de Certeau, La fable mystique, Bd. 2 (Anm. 7), 337-357.

${ }_{11}$ Vgl.Jean-Jacques Courtine, Une anthropologie de la voix, in: Delacroix et al. (Hg.), Michel de Certeau, 179-189, 182. Dazu gehörten unter anderen auch die Psycholinguisten Jean Bobon und André Roch-Lecours, die Certeau in seinem Aufsatz erwähnt.

12 Langages 91: Les glossolalies (1988).

${ }^{13}$ Michel de Certeau,Vocal Utopias: Glossolalias, in: Representations 56 (1996), 2947.
} 
bereich weit offen hält. So steht die Glossolalie der vernünftigen Sprache gegenüber als das Unsinnige schlechthin - und wird von dieser wegen ihrer Unverständlichkeit durch alle möglichen Kategorisierungen degradiert und handhabbar zu machen versucht. Diesen Verfahren, mit denen die rationale wissenschaftliche Aneignung sich das Unsinnige zu eigen machen will, indem sie es erklärt und ihre Bedeutung fixiert, spürt Certeau sorgfältig und kritisch nach. Er legt dabei offen, wie die vernünftige Sprache ihr Gegenteil, den Unsinn, als ihre eigene Bedingung in sich trägt - und auch die Zungenrede auf den Sinn als ihr Anderes angewiesen ist. Beide "Positionen der Sprache gehören zusammen wie Zwillinge oder eben wie eine Bühne und der Raum hinter den Kulissen, der doch entscheidend mitbestimmt, was sich vorne auf der Bühne abspielt.

Über den Begriff der Institution verfolgt er diese Dynamik der zwiefältigen Stiftung von Sprache (als reines Sagen und als ein etwas-Sagen) bis in die Konventionen der Sprache und der damit verbundenen Frage nach Macht in unserer Gesellschaft hinein. Insgesamt liest sich seine Beschreibung wie ein Plädoyer für das »Gras zwischen den Pflastersteinen«.

\section{Die Übersetzung}

Generell haben wir versucht bei der Übersetzung so nah wie möglich am Französischen zu bleiben und die zum Teil eigenwillige und elliptische Ausdrucksweise des Autors ${ }^{14}$ nicht zu sehr zu glätten. Zwischen diesem Ziel und einer grösstmöglichen Verständlichkeit galt es immer wieder die Mitte zu finden. So liess sich beispielsweise die einheitliche Übersetzung von parler (sprechen) und dire (sagen) oder auch von semblant (das Ähnliche, der Anschein) und discours (die Rede, der Diskurs) nicht durchhalten. Das liegt auch daran, dass Certeau die Worte in ihrer Nuancenvielfalt, die sich aus den unterschiedlichen Disziplinen (Linguistik, Psychoanalyse, Theologie usw.) speist, auslotet, in Spannung zueinander setzt und mit ihr spielt. Deswegen haben wir jeweils das Original in Klammern dazu geschrieben, wenn es uns nötig erschien.

Deutsche oder andere fremdsprachige Zitate im Fliesstext und den Fussnoten haben wir, soweit sie zu finden waren, direkt aus

\footnotetext{
${ }^{14}$ Vgl. dazu Füssel, Einleitung, 13: "Der an Metaphern reiche Stil Certeaus verleiht seinen Schriften eine literarische Qualität, die sicher einen Grossteil der von seinem Werk ausgehenden Faszination ausmacht. Andererseits wird dadurch Verständnis und Darstellbarkeit seiner Argumentation nicht immer erleichtert."
} 
den Originalausgaben bzw. Ausgaben der deutschen Übersetzungen wiedergegeben und ansonsten selbständig übersetzt. Allfällige Korrekturen wurden dabei von uns stillschweigend vorgenommen. Die Kursivsetzung folgt weitestgehend dem französischen Text, nur die lateinischen Ausdrücke wurden von uns zusätzlich kursiv gesetzt.

- Dipl. theol. Johanna Breidenbach ist Assistentin am Lehrstuhl für Systematische Theologie an der Theologischen Fakultät der Universität Zürich.

- Dr. theol., Dr. sc. rel. Lucie Kaennel ist wissenschaftliche Assistentin am Lehrstuhl für Systematische Theologie, insbesondere Hermeneutik und Fundamentaltheologie, an der Theologischen Fakultät der Universität Zürich. 